\title{
A detailed study of FDIRC prototype with waveform digitizing electronics in cosmic ray telescope using 3D tracks
}

\author{
K. Nishimura ${ }^{b}$, B. Dey ${ }^{\text {e }}$, D. Aston ${ }^{\text {c }}$, D.W.G.S. Leith ${ }^{c}$, B. Ratcliff ${ }^{c}$, D.Roberts ${ }^{\mathrm{d}}$, \\ L.Ruckman ${ }^{\text {b }}$, D.Shtol ${ }^{\text {a }}$, G.S. Varner ${ }^{\text {b }}$, J. Va'vra ${ }^{\text {c,* }}$ \\ ${ }^{a}$ Budker Institute Of Nuclear Physics, Novosibirsk, Russia \\ ${ }^{b}$ University of Hawaii, Honolulu, HI 96822, USA \\ ${ }^{c}$ SLAC, Stanford University, CA 94309, U.S.A. ${ }^{* *}$ \\ ${ }^{d}$ University of Maryland, U.S.A. \\ ${ }^{e}$ University of California, Riverside, CA 92521, U.S.A. \\ Elsevier use only: Received date here; revised date here; accepted date here
}

\begin{abstract}
We present a detailed study of a novel Cherenkov imaging detector called the Focusing DIRC (FDIRC) with waveform digitizing electronics. In this test study, the FDIRC prototype has been instrumented with seven Hamamatsu H-8500 MaPMTs. Waveforms from $\sim 450$ pixels are digitized with waveform sampling electronics based on the BLAB2 ASIC, operating at a sampling speed of $\sim 2.5$ $\mathrm{GSa} / \mathrm{s}$. The FDIRC prototype was tested in a large cosmic ray telescope (CRT) providing 3D muon tracks with $\sim 1.5$ mrad angular resolution and muon energy of $E_{\text {muon }}>1.6 \mathrm{GeV}$. In this study we provide a detailed analysis of the tails in the Cherenkov angle distribution as a function of various variables, compare experimental results with simulation, and identify the major contributions to the tails. We demonstrate that to see the full impact of these tails on the Cherenkov angle resolution, it is crucial to use 3D tracks, and have a full understanding of the role of ambiguities. These issues could not be fully explored in previous FDIRC studies where the beam was perpendicular to the quartz radiator bars. This work is relevant for the final FDIRC prototype of the PID detector at SuperB, which will be tested this year in the CRT setup.
\end{abstract}

\footnotetext{
* Corresponding author. Tel.: J. Va’vra, 650-926-2658; fax: 650-926-4178; e-mail: jjv@slac.stanford.edu.

${ }_{* *}^{*}$ Work has been supported in part by the Department of Energy Advanced Detector Research Program Award \# DE-FG02-08ER41571

Work supported by the Department of Energy, contract DEAC02-76SF00515.
} 


\section{Introduction}

The DIRC detector at the BaBar experiment provided excellent particle identification performance $[1,2]$. Based on this success, our group has been following an R\&D program to develop a compact and fast detector for future particle identification systems. Our initial attempt was to build the first FDIRC prototype [3-7], which was capable of measuring $\mathrm{x}-\mathrm{y}$ coordinates for each photon with an angular resolution similar to the present BaBar DIRC, but, in addition, could also measure the time-of-propagation $\left(\mathrm{TOP}^{1}\right)$ of each photon along the fused silica bar with a $200-250$ ps single-photoelectron timing resolution (the present BaBar DIRC has a timing resolution of only $\sigma$ $\sim 1.6 \mathrm{~ns}$ ). The first FDIRC prototype had a successful SLAC test beam run. It was the first RICH detector to demonstrate correction of chromatic error by timing [5-7]. ${ }^{1}$ In these initial tests we used Elantek 2075 amplifiers with a gain of 40x, and custom constant-fraction discriminators (CFD) coupled to a 25 ps/count Philips 7186 TDC. These initial tests concentrated on proving the principle of focusing optics, learning to operate highly pixilated fast detectors, and showing that the chromatic broadening can be corrected by timing.

In this paper we present a study of the second FDIRC prototype, which has the same optics as the first prototype, but incorporates a different set of detectors and electronics; we used six 64-pixel H8500 MaPMTs, which were read out by the highly integrated electronics based on the BLAB2 ASIC [8$13]$.

We concentrate on better understanding of the Cherenkov angle resolution tails, which were never fully understood in earlier studies [14].

The second prototype was tested in a large Cosmic Ray Telescope (CRT) [15] capable of providing 3Dtracking with $\sim 1.5 \mathrm{mrad}$ resolution with hard muons $\left(\mathrm{E}_{\text {muon }}>1.6 \mathrm{GeV}\right)$. CRT tests now serve as an excellent test-bed for developing various FDIRC concepts.

\footnotetext{
1 The red photons arrive before blue photons by $\sim 1.5 \mathrm{~ns}$ in a $3.6 \mathrm{~m}$-long FDIRC bar, and therefore they can be tagged by timing, if one achieves a timing resolution of $\sim 200 \mathrm{ps} /$ photon; such timing correction can reduce the Cherenkov angle resolution by $\sim 0.5 \mathrm{mrad} /$ photon from $9.5 \mathrm{mrad} /$ photon [5-7].
}

One should point out that a newer FDIRC detector scheme was designed more recently, based on solid Fused Silica optics; we call it the final FDIRC prototype. The overall aim of the new design is to have a photon camera (equivalent to the BaBar DIRC Stand-Off-Box) approximately $\sim 25 \mathrm{x}$ smaller and $10 \mathrm{x}$ faster than the BaBar DIRC in order to reduce sensitivity to the higher background expected at SuperB [16].

\section{Description of the FDIRC prototype}

Figure 1a shows the concept and practical realization of the second FDIRC prototype (Fig.1b). This prototype consists of a single DIRC bar (Fig. 1c) of $\sim 3.6 \mathrm{~m}$ length $(1.7 \mathrm{~cm}$ thick and $3.5 \mathrm{~cm}$ wide), a focusing element made of a $50 \mathrm{~cm}$ focal length spherical mirror (Fig.1d) placed in a small optical box filled with mineral oil, ${ }^{2}$ which serves as the coupling medium between the bar and the focal plane (Fig.1e). The spherical mirror is designed to remove the effect of bar thickness on the resolution since parallel rays are focussed to the same point on the detector plane. The focal plane (Fig.1f) has seven 64pixel Hamamatsu Flat-panel H-8500 MaPMT photon detectors. We use a PiLas laser ${ }^{3}$ for calibration. Figure 1a shows the laser entry into the optics.

The BLAB2 waveform digitizing electronics [8-13] was operated at a sampling rate of $\sim 2.5 \mathrm{GSa} / \mathrm{s}$. Control and readout is done via Gigabit fiber-optic transceivers connected to a compact-PCI readout crate with an embedded CPU running Linux. We operate the photo-detectors at $\sim 10^{6}$ gain. An onASIC trans-impedance gain of about $2 \mathrm{k} \Omega(\sim 40 \mathrm{x}$ voltage gain) was a design goal. Figure 2 shows a few details of the BLAB2 electronics implementation on this prototype. In total we installed electronics for 384 pixels. As seen in Fig.2b, the BLAB-based readout modules have been installed on six of the $\mathrm{H}$ 8500 MaPMTs, and the rightmost slot has been left instrumented by an existing Elantek amplifier to provide analog monitoring. Figure $2 \mathrm{c}$ shows an example of single photo-electron pulse produced by the BLAB2 waveform digitizing electronics.

The BLAB2 electronics achieved a timing

\footnotetext{
${ }^{2}$ KamLand experiment mineral oil: Bicron BC-599-14.

${ }^{3}$ Manufactured by Advanced Laser Diode Systems, D-12489 Berlin, Germany.
} 
resolution of only 1-2 ns in CRT tests, which did not allow for performing chromatic corrections. However, due to the robustness of the proposed FDIRC concept, we could still perform many other useful studies that did not require high resolution timing.

(a)

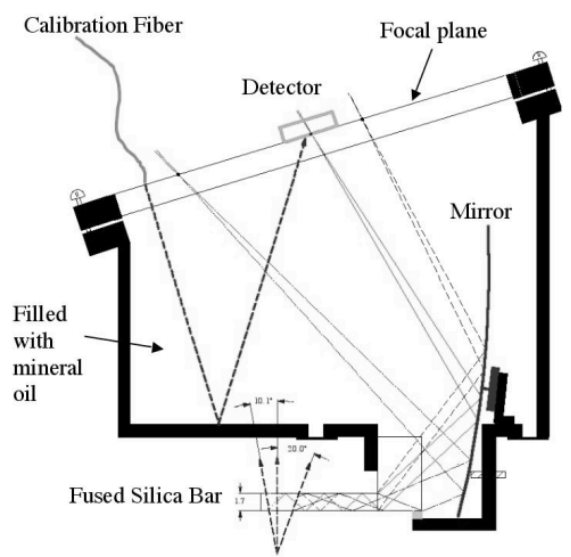

(b)

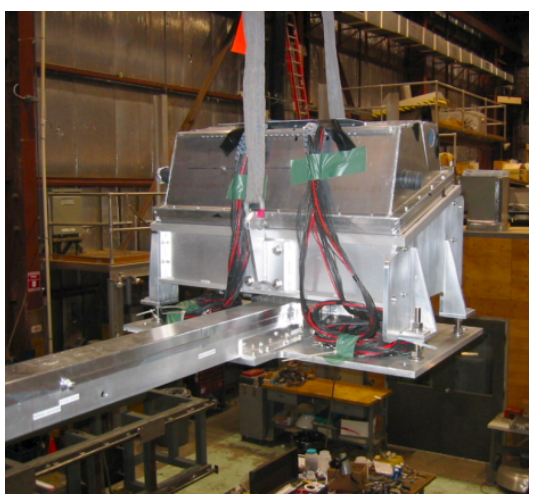

(c)

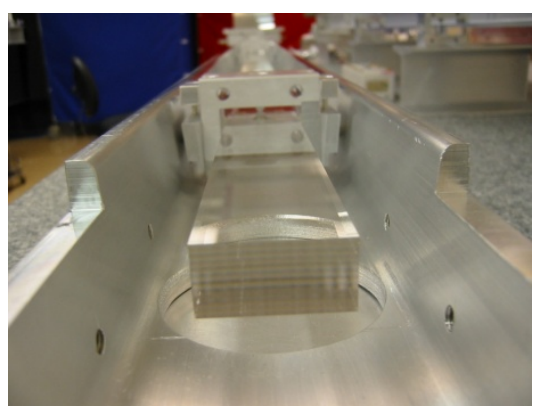

(d)

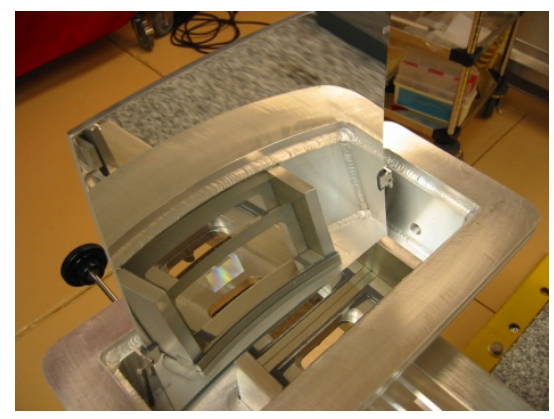

(e)

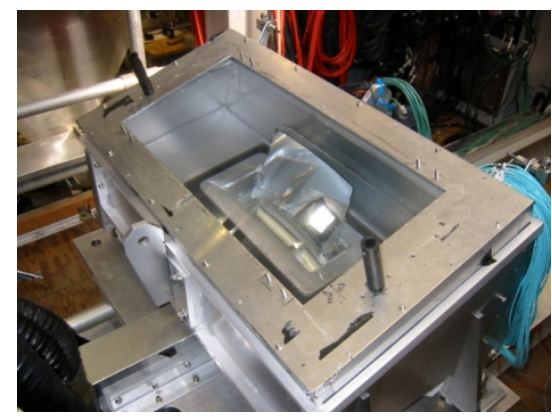

(f)

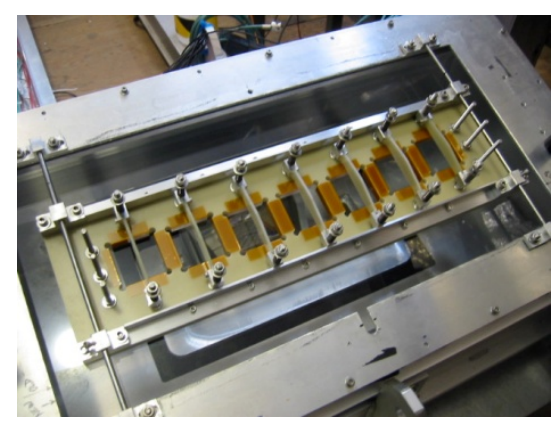

Fig. 1. (a) Principle of the first FDIRC prototype used in Refs. 3-7 and 9. (b) The FDIRC prototype used for the study in this paper with photon camera and single $3.6 \mathrm{~m}$ long bar. (c) Fused Silica radiator bar $(1.7 \mathrm{~cm}$-thick $\mathrm{x}$ $3.5 \mathrm{~cm}$-wide $\mathrm{x} 3.6 \mathrm{~m}$-long), (d) Spherical lens in the photon camera. (e) Photon camera with a spherical lens and filled with Kamland oil. (f) H-8500 detector holder with 7 available slots. 
(a)

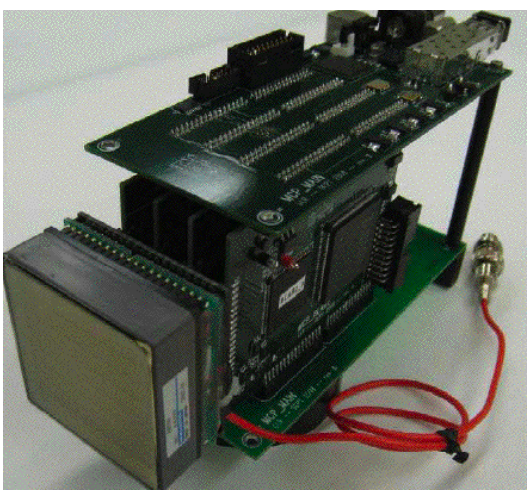

(b)

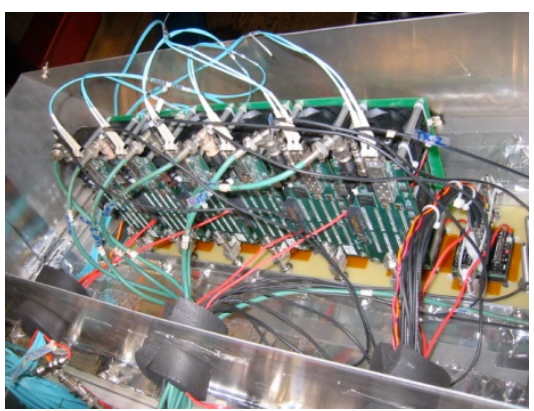

(c)

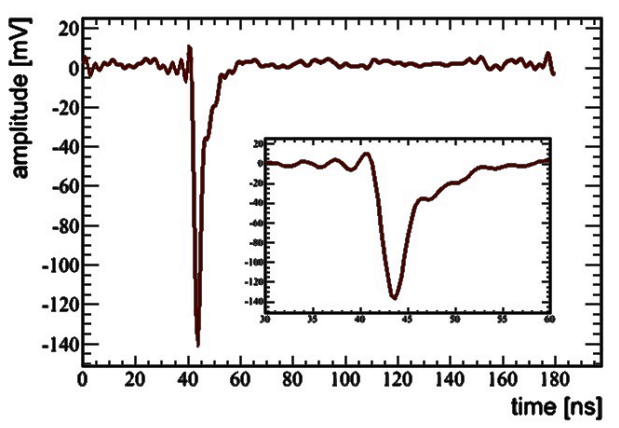

Fig. 2. (a) BLAB2 electronics package integrated with an $\mathrm{H}-8500$ MaPMT. (b) Six BLAB2 packages installed in the prototype, together with one Elantek-based amplifier package (on the right), used to set up timing and provide analog monitoring. (c) Example of a single electron waveform from BLAB2.

\section{Cosmic Ray Telescope (CRT)}

We have constructed a large cosmic ray telescope at SLAC (see Fig. 3, and Ref. 8 for more detailed description). The telescope consists of upper and lower orthogonal planes of scintillator-based hodoscope tracking, providing $\sim 1-1.5 \mathrm{mrad}$ angular tracking resolution, and a stack of four $\sim 30 \mathrm{~cm}$-thick iron absorber plates with interleaved scintillator counters S1-S4, providing muon energy selection. The trigger is formed from a coincidence of two large-size scintillators $\mathrm{T} 1 \& \mathrm{~T} 2$ and a "bar footprintdefining” Quartz start counter (Qtz). The quartz counter with an MCP-PMT readout provides a precision start time. ${ }^{4}$ Measuring the muon range in the iron stack provides a coarse energy selection (the largest cut-off energy, requiring the S1 counter to fire, is $>1.6 \mathrm{GeV})$. In this configuration and with $\mathrm{T} 1 * \mathrm{~T} 2 * \mathrm{Q} t z$ trigger, we obtained typically $\sim 6 \mathrm{k}$ of "hard $>1.6 \mathrm{GeV}$ muon tracks" per day.

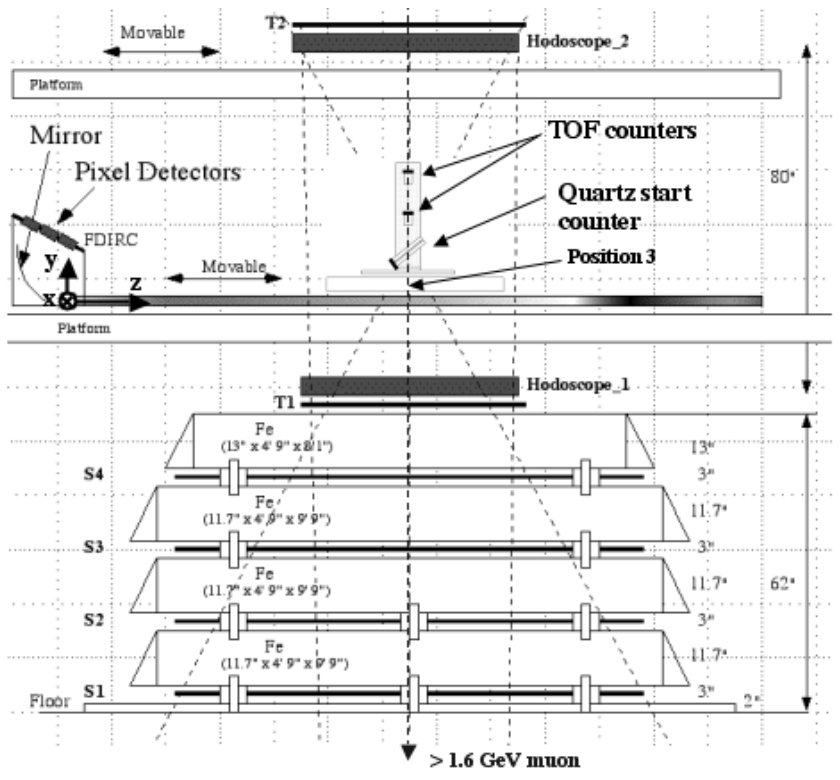

Fig.3. Schematic drawing of the the second FDIRC prototype located in a large cosmic ray telescope built at SLAC [15]. The positions shown correspond to the so called "nominal" hodoscope \& trigger counter (x-axis goes into the page, $y$-axis up, and $\mathrm{z}$-axis along the bar).

\section{Experimental results in CRT}

This analysis is based on a total sample of $\sim 370 \mathrm{k}$ CRT triggers $(\mathrm{T} 1 * \mathrm{~T} 2 * \mathrm{Qtz})$, which were collected

\footnotetext{
${ }^{4}$ In the test beam we routinely obtained a resolution of $\sim 42 \mathrm{ps}$ for the start time. With 3D tracks in the CRT setup, integrating over long running time periods, we achieved $\sim 70 \mathrm{ps}$.
} 
over a period of several months. Of these, approximately $\sim 72 \mathrm{k}$ were good single tracks with $\mathrm{E}_{\text {muon }}>1.6 \mathrm{GeV}$, suitable for analysis.

\section{a. Time and amplitude extraction}

Before a system trigger is received, the BLAB2 waveform digitizers sample continuously into an analog storage buffer with a depth of 3072 samples, corresponding to $\sim 1.2 \mu \mathrm{s}$ of storage at $2.5 \mathrm{GSa} / \mathrm{s}$. Individual comparators in each BLAB2 channel are used to monitor for photon hits, and the timing of these comparator signals is used to flag regions of interest within the analog storage array. Upon receipt of the system trigger, signals from 512 samples of each channel, specific to previously defined regions of interest, are digitized and read out. A 17th channel on each BLAB2 is used to digitize the NIM-level system trigger signal, which is used to globally align the time from different ASICs, each of which runs on an independent sampling clock.

Raw waveforms for each event are stored to disk to allow for offline signal processing analysis. During this offline analysis, the following steps are performed to calculate the time and signal-size of each photon hit:

1) Pedestal voltages are subtracted from each waveform. This removes fixed DC offsets that vary from sample-to-sample in the BLAB2.

2) Any remaining common DC offset in each waveform is removed by measuring the average waveform value before the pulse arrival and subtracting it from all samples.

3) Waveforms from signal channels are digitally processed with a $300 \mathrm{MHz}$ low-pass filter. This filter mitigates the effects of a sinusoidal oscillation seen in the on-chip BLAB2 amplifier. This step is not necessary for the system trigger waveform.

4) Timing for signal channels and the system trigger channel is determined through a software implementation of a constant-fraction discriminator. The minimum waveform value is first determined by scanning through the waveform points. Then a scan is performed to locate the time when $30 \%$ of this value is reached. A linear interpolation is used when this point falls between two samples.

5) The globally aligned time for each hit is determined using the relative timing between the digitized system trigger and the digitized PMT signal.

6) The total charge of the hit is estimated using a simple rectangular numerical integration for each PMT waveform, assuming a $2 \mathrm{k} \Omega$ load resistance, consistent with that of the BLAB2 internal amplifier.

The final timing resolution obtained with this technique is limited to order $1-2 \mathrm{~ns},{ }^{5}$ both due to effects of the amplifier oscillation and subsequent filtering, and the relatively poor synchronization provided by the trigger digitization scheme. A global clock scheme is preferred to guarantee synchronous sampling between all ASICs, and is being implemented for the final FDIRC prototype.

\section{b. Introduction to the FDIRC data analysis}

We define a coordinate system as described in Figs. 3 and 4.

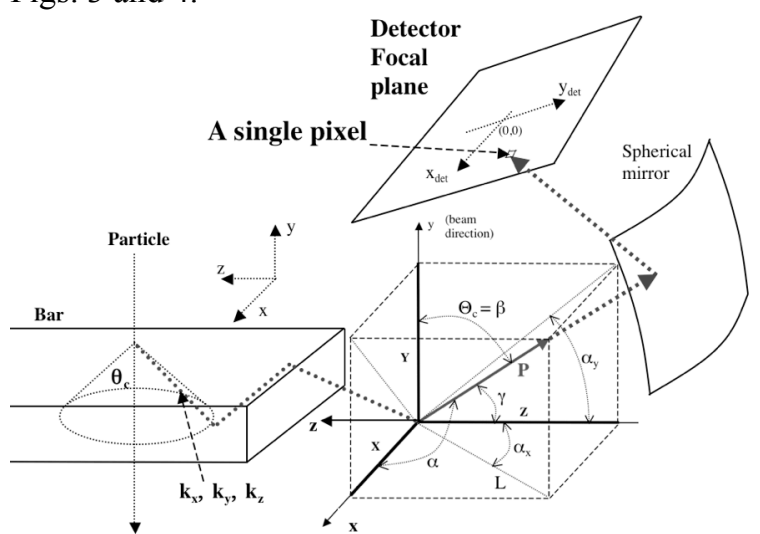

Fig.4. The FDIRC prototype coordinate system defining various variables used in this paper.

In DIRC-like detectors, each detector pixel gets a unique assignment of $\mathrm{k}_{\mathrm{x}}, \mathrm{k}_{\mathrm{y}}$ and $\mathrm{k}_{\mathrm{z}}$ direction cosines. The pixel-based constant assignments are determined from the MC simulation generating randomly distributed photons at the bar center, and propagating them along the bar to the detector end and then to a given detector pixel. One then forms an average photon vector assignment for each pixel: $\mathbf{k}_{\text {photon }}=\left(\mathrm{k}_{\mathrm{x}}\right.$, $\mathrm{k}_{\mathrm{y}}, \mathrm{k}_{\mathrm{z}}$ ) - we call these constants "reconstructed" (as

\footnotetext{
${ }^{5}$ Bench tests with BLAB2 electronics achieved a timing resolution of $\sim 0.5 \mathrm{~ns}$. However, tests in CRT achieved a dTOP resolution of only $\sim 1.9 \mathrm{~ns}$.
} 
opposed to "true" constants, which can be generated only by MC and correspond to the origin of photon production). Only two of the direction cosines are measured and the third is deduced from the sum of the squares. Constants are determined up to a sign (see further discussion). Once these constants are determined, the data analysis consists of looping over all pixels with hits, and calculating derived quantities such as those shown in this section, i.e., number of bounces in the bar, time-of-propagation, photon path length and the Cherenkov angle. The CRT tracking provides the required information on the position of the muon track in the bar $\left(\mathrm{z}_{\text {particle }}\right)$ and track direction $\left(\mathbf{k}_{\text {track }}\right)$. It is useful to review several derived quantities we will need in our discussion. With the help of Fig. 4, we can write photon direction cosines in the bar coordinate system as:

$$
\mathrm{k}_{\mathrm{x}}=\cos \alpha, \mathrm{k}_{\mathrm{y}}=\cos \beta, \mathrm{k}_{\mathrm{z}}=-\cos \gamma
$$

The photon path length in the bar for photons propagating towards the detector:

$$
\mathrm{L}_{\text {path }}(\text { direct photons })=\mathrm{z}_{\text {particle_position }} / \mathrm{k}_{\mathrm{z}} \text {, }
$$

where $z_{\text {particle_position }}$ is determined from a track's intersection with the bar. The photon path length in the bar for photons propagating towards the mirror located at bar's end:

$$
\left.\mathrm{L}_{\text {path }} \text { (indirect photons }\right)=\left(2 \mathrm{~L}_{\text {bar }}-\mathrm{Z}_{\text {particle_position }}\right) / \mathrm{k}_{\mathrm{z}}
$$

The time-of-propagation in the bar from a track entry point $\left(\mathrm{v}_{\mathrm{g}}(\lambda)\right.$ being the group velocity and $\mathrm{n}_{\mathrm{g}}(\lambda)$ the group refractive index):

$$
\begin{aligned}
\operatorname{TOP}(\lambda) & =\mathrm{L}_{\text {path }} / \mathrm{v}_{\mathrm{g}}(\lambda)=\mathrm{L}_{\text {path }} * \mathrm{n}_{\mathrm{g}}(\lambda) / \mathrm{c} \\
& =\mathrm{L}_{\text {bar }} * \mathrm{n}_{\mathrm{g}} /\left(\mathrm{k}_{\mathrm{z}} \mathrm{c}\right)
\end{aligned}
$$

The photon's group velocity is

$$
\mathrm{v}_{\mathrm{g}}(\lambda)=\mathrm{c} / \mathrm{n}_{\mathrm{g}}(\lambda)=\mathrm{c} /\left[\mathrm{n}_{\text {phase }}-\lambda * \mathrm{dn}_{\text {phase }} / \mathrm{d} \lambda\right],
$$

where $\mathrm{c}$ is the velocity of light in vacuum, $\mathrm{n}_{\text {phase }}$ is the phase refractive index of the Fused Silica and $\lambda$ is the photon wavelength. Another quantity we will need in our analysis is the number of total photon bounces in the bar

$$
\begin{gathered}
\mathrm{n}_{\text {bounces }}=\mathrm{n}_{\mathrm{x}}+\mathrm{n}_{\mathrm{y}}=\mathrm{L}_{\text {path }} /\left[\operatorname{bar}_{\text {width }} * \operatorname{abs}\left(\mathrm{k}_{\mathrm{z}} / \mathrm{k}_{\mathrm{x}}\right)\right]+ \\
\mathrm{L}_{\text {path }} /\left[\operatorname{bar}_{\text {height }} * \operatorname{abs}\left(\mathrm{k}_{\mathrm{z}} / \mathrm{k}_{\mathrm{y}}\right)\right],
\end{gathered}
$$

where $n_{x}$ is the number of bounces from the bar's side, and $\mathrm{n}_{\mathrm{y}}$ is the number of bounces from the bar's face.

Using 3D tracks from the CRT, the Cherenkov angle is easily obtained as the dot product of two vectors:

$$
\cos \theta_{\mathrm{c}}=\mathbf{k}_{\text {photon }} \cdot \mathbf{k}_{\text {track }},
$$

where $\mathbf{k}_{\text {track }}$ is the track direction vector obtained from the CRT tracking, and $\mathbf{k}_{\text {photon }}$ is the photon vector $\left(\mathrm{k}_{\mathrm{x}}, \mathrm{k}_{\mathrm{y}}, \mathrm{k}_{\mathrm{z}}\right)$ obtained from the pixel assignment from the MC program.

\section{c. Reach of some critical variables}

Figure 5 shows a typical range of track angles, $\theta_{\text {track }}$ and $\phi_{\text {track}}$, defined in the spherical coordinate system relative to the z-axis, for the nominal position of the CRT hodoscopes \& trigger counters. The track angular range is about $\pm 20^{\circ}$ in $\theta_{\text {track }}$ and $\pm 8^{\circ}$ in $\phi_{\text {track }}$. The $\theta_{\text {track }}$ range for good Cherenkov ring reconstruction gets reduced further to about $\pm 5^{\circ}$ because of our limited detector coverage. Although the range of these variables is relatively small, it is nevertheless necessary to perform the 3D-track treatment correctly in order to achieve a good Cherenkov angle resolution.

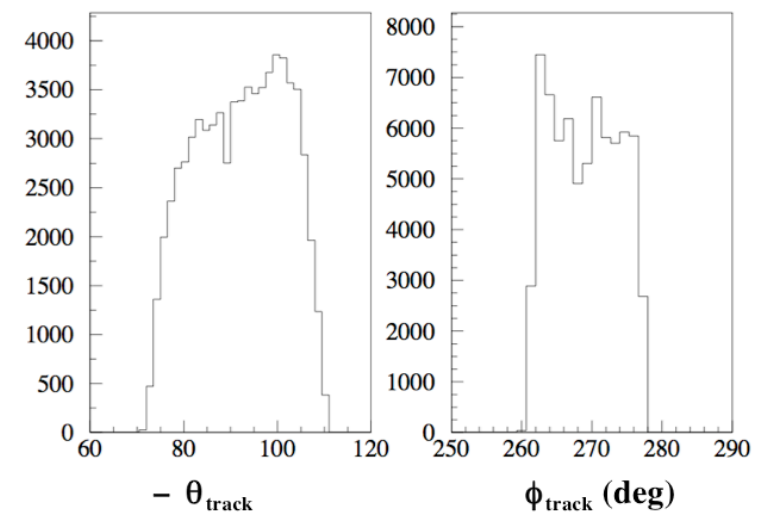

Fig.5. Typical measured range in this analysis of track angular variables $\theta_{\text {track }}, \phi_{\text {track }}$ for the nominal hodoscope $\&$ trigger counter positions in CRT - see Fig. 3. 

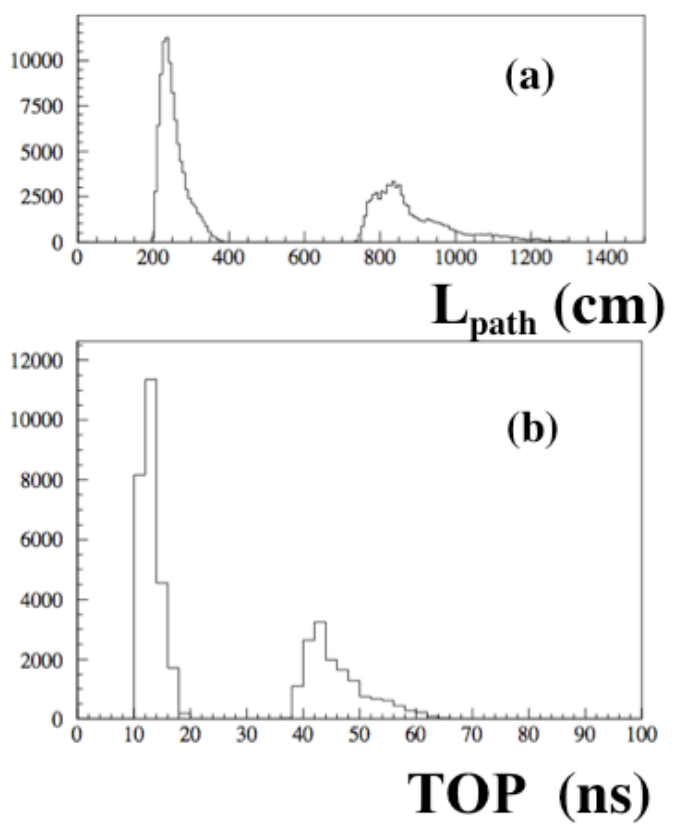

Fig.6. (a) Measured distributions of the photon path length in the bar $\mathrm{L}_{\text {path }}$ and (b) time-of-propagation, TOP, for the nominal CRT setup (TOP $<30$ ns defines direct photons, and TOP $>30 \mathrm{~ns}$ defines indirect photons).

Figure 6 shows the $\mathrm{L}_{\text {path }}$ and TOP distributions, again in the nominal CRT setup, for both direct and indirect photons. In the prototype configuration utilized for this study, direct and indirect photons typically traveled 2-3 meters and 8-12 meters, respectively, with TOP values ranging between 10 and $20 \mathrm{~ns}$, while indirect photons 8-12 meters, with TOP values ranging between 35 and $65 \mathrm{~ns}$. One can also see that direct and indirect photons can be easily separated by a simple timing cut. Figure $6 b$ shows how this is done.

Figure 7 shows the dependence of the photon path length in the bar $\left(\mathrm{L}_{\mathrm{path}}\right)$ on the track direction cosines $\mathrm{k}_{\mathrm{x}}, \mathrm{k}_{\mathrm{y}}$, and $\mathrm{k}_{\mathrm{z}}$ for the nominal CRT setup.
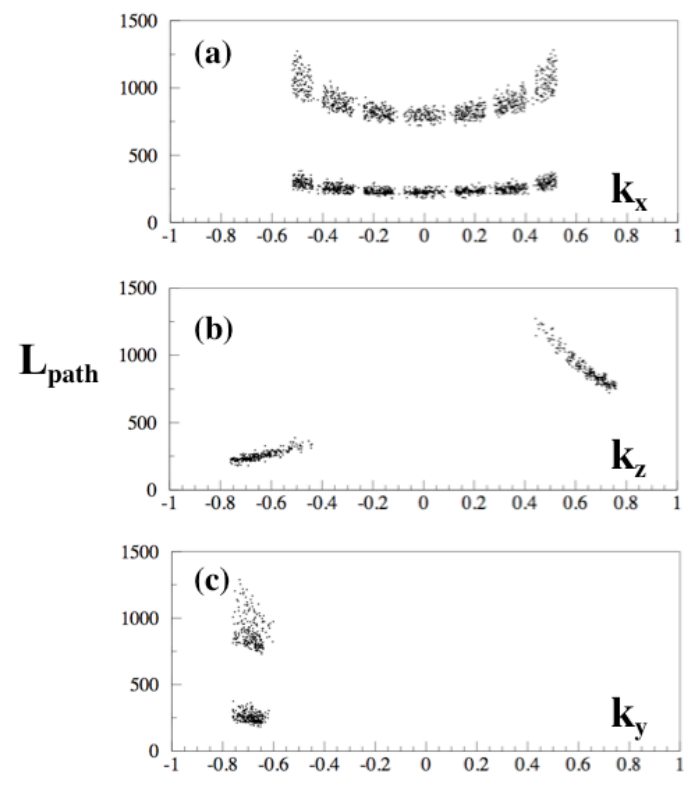

Fig.7. Measured photon path length $\mathrm{L}_{\text {path }}$ as a function of track direction cosines (a) $\mathrm{k}_{\mathrm{x}}$, (b) $\mathrm{k}_{\mathrm{z}}$, and (c) $\mathrm{k}_{\mathrm{y}}$ for the nominal CRT setup.

Figures $8 \mathrm{a}$ and $\mathrm{b}$ show the total number of photon bounces $\mathrm{n}_{\text {total }}=\mathrm{n}_{\mathrm{x}}+\mathrm{n}_{\mathrm{y}}$, and the correlation between $\mathrm{n}_{\mathrm{x}}$ and $\mathrm{n}_{\mathrm{y}}$ for all photons, direct and indirect. One can see that one deals with a very large number of photon bounces in the bar, approaching $\sim 1200$ in this setup, and that the number of bounces in the $\mathrm{x}$-direction and $y$-direction correlate with each other in a rather complicated way. Figure $8 \mathrm{c}$ shows the correlation between the detector slot number and the number of bounces $\mathrm{n}_{\mathrm{x}}$, for all photons, direct and indirect. One can see that slot 4 (corresponding to the central detector - see Fig. 2f) is hit only if the number of side bounces is small $\left(\mathrm{n}_{\mathrm{x}}<30\right)$, i.e., photons going into this slot bounce mainly from bar faces $\left(\mathrm{n}_{\mathrm{y}}\right.$ is actually large for this case - see Fig. 8b). We will see later that the central portion of the Cherenkov ring has the best resolution in this detector. 

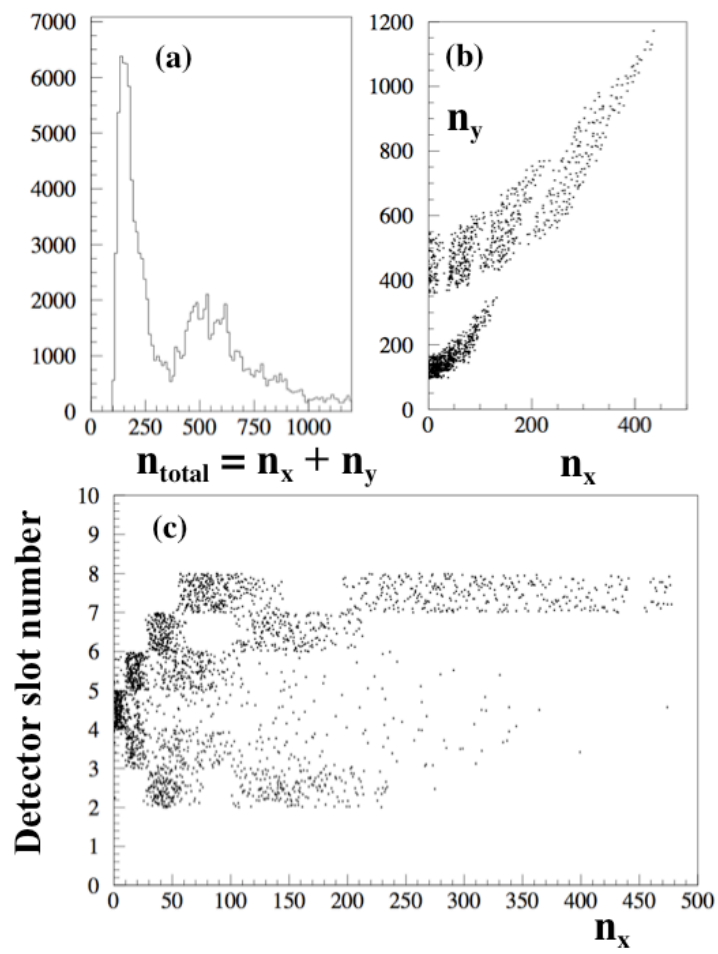

Fig.8. (a) Measured total number of photon bounces in the bar $\mathrm{n}_{\text {total }}=\mathrm{n}_{\mathrm{x}}+\mathrm{n}_{\mathrm{y}}$; (b) correlation between $\mathrm{n}_{\mathrm{x}}$ and $\mathrm{n}_{\mathrm{y}}$, and; (c) correlation between the detector slot number and the number of bounces $n_{x}$, for all photons, direct and indirect.

\section{Cherenkov angle distribution and tails}

It is useful to summarize the Cherenkov angle resolution as measured by the BaBar DIRC, the first FDIRC prototype in the test beam, and the second prototype in the CRT setup. Although we list these results here, we warn that direct comparisons of Cherenkov angle resolutions and performances are not entirely comparable since the electronics, experimental conditions, detector coverage, and analysis methods all differ.

\section{a. BaBar DIRC}

The BaBar DIRC had full detector coverage, of course. Figure 9 shows the Cherenkov angle distribution as measured in the BaBar experiment using di-muon events [1], and comparison to a MC simulation [14]. Figure 9a shows that there is a broad background of less than $10 \%$ relative height under the peak, that originates mostly from track-associated sources, such as $\delta$-rays, and combinatorial background. The width of the peak translates to a resolution of about $9.6 \mathrm{mrad}$, in good agreement with the expected value.
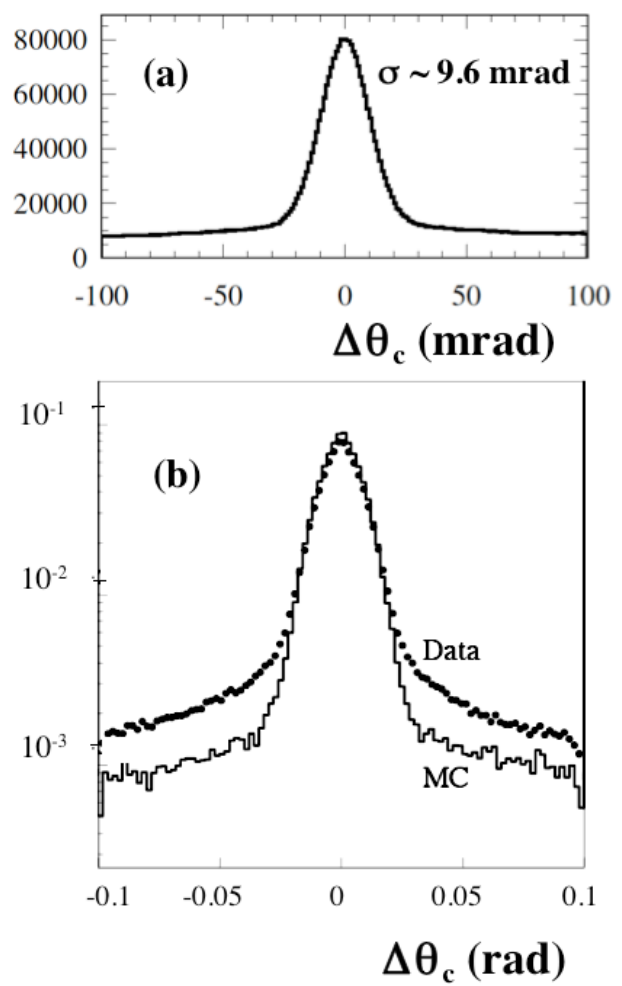

Fig.9. (a) Distribution of $\Delta \theta_{c}=\left(\theta_{c}-\theta_{\text {mean }}\right)$ for $\mathrm{e}^{+} \mathrm{e}^{-}$ $\rightarrow \mu^{+} \mu^{-}$pairs as reconstructed in the BaBar DIRC [1]. (b) Comparison of the same data (dots) and the Monte Carlo prediction using the "official" DIRC MC program, which includes delta rays [14].

Figure $9 \mathrm{~b}$ shows that the MC prediction gives the same resolution as data, but the tail amplitudes differ [14]. There was a considerable experimental and MC simulation effort to explain this discrepancy. For example, a detailed dedicated experimental study attempted to explain this discrepancy by scintillation in the fused silica bar when a particle passes through [14]. It was determined that the scintillation mechanism is negligible and represents less than $1 \%$ of the total photon background. Instead, it was found that there are two other major components in the DIRC photon background. One 
component consists of photons created by the $\delta$-ray electrons in the fused silica, which in turn produce Cherenkov light (this effect was already included in DIRC MC simulation). The second component is caused by the reflection of photons at the EPOTEK301-2 glue/fused silica interfaces due to a small difference in refractive indices (this effect was added to the DIRC MC simulation after this test). However, even after the glue reflection effect was properly included in the BaBar MC simulation, there was still a discrepancy similar to that shown in Fig. 9b, because the glue reflection effect was too small.

\section{b. The first FDIRC prototype in the SLAC test beam}

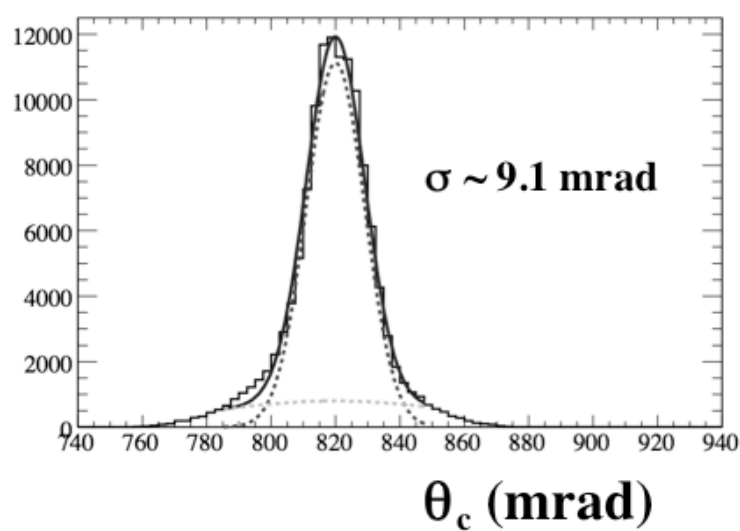

Fig.10. Measured Cherenkov angle distribution in the first FDIRC prototype in the SLAC $10 \mathrm{GeV} / \mathrm{c}$ electron test beam (evaluated for indirect photons with a photon path length of $\mathrm{L}_{\text {path }} \sim 10 \mathrm{~m}$ ). Tracks entered perpendicularly to the bar face.

This particular FDIRC prototype had limited detector coverage, matched exactly to the ring images obtained with beam perpendicular to the bar. These are results with the FDIRC prototype equipped with SLAC Amp/CFD/TDC electronics and taking data in the SLAC $10 \mathrm{GeV} / \mathrm{c}$ electron test beam [5-7]. Figure 10 shows the measured Cherenkov angle distribution. The data analysis uses all detector slots and only indirect photons traveling more than $10 \mathrm{~m}$ in the bar, so a chromatic correction was applied. Because the detector coverage is not as complete as in the BaBar DIRC, the observed tail is not flat as in Fig. 9a; instead, it is effectively cut at $\pm 60 \mathrm{mrad}$ relative to the mean. Nevertheless, we believe that there is a comparable level of combinatorial background under the peak as that observed in the BaBar DIRC.

\section{c. The second FDIRC prototype in the CRT}

The second FDIRC prototype [8] also had limited detector coverage. However, because the tracks are no longer only perpendicular to the bar, as was the case in the test beam, some ring images were outside the detector acceptance. To see good Cherenkov angle resolution in the CRT, one has to deal with the 3D track algorithm, as described in section $4 \mathrm{~b}$.

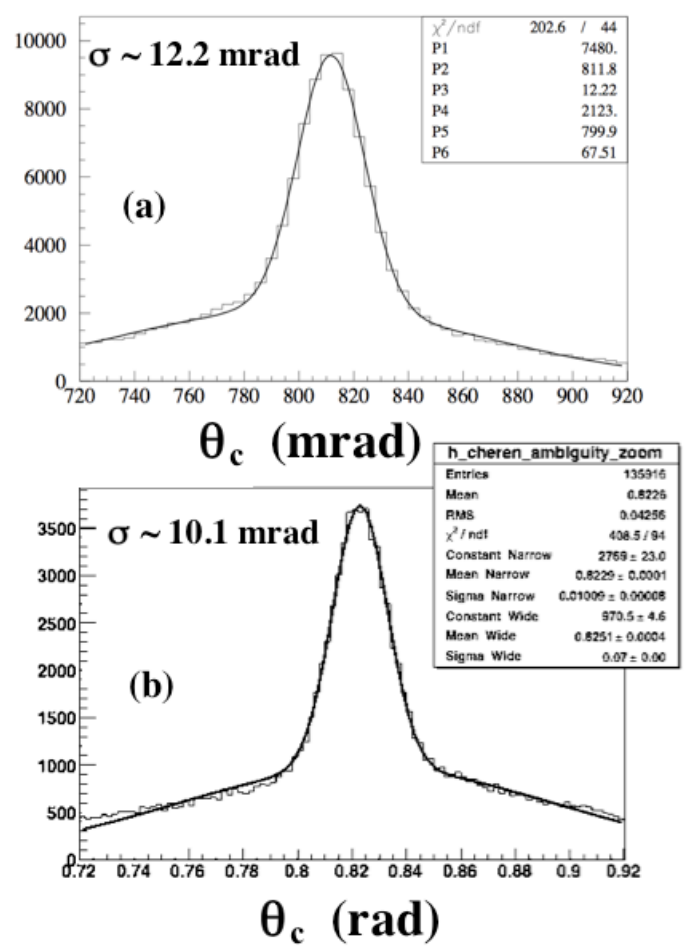

Fig.11. (a) Measured Cherenkov angle distribution for $\mathrm{E}_{\text {muon }}>1.6 \mathrm{GeV}$ in the second FDIRC prototype in CRT. (b) The corresponding $\mathrm{MC}$ simulation under the same conditions. Tracks entered the bar face with angles shown on Fig. 5, i.e., we are dealing with 3D tracks.

Figure 11 shows measured and simulated Cherenkov angle distributions with no special cuts. Again, the tail is affected by limited detector coverage in the prototype; therefore we do not see a flat background as in Fig. 9a. The measured resolution is 
$\sigma \sim 12.2 \mathrm{mrad}$ per photon, which is somewhat larger than the MC simulation expectation $(\sigma \sim 10.1 \mathrm{mrad})$. This can be explained by the fact that in the CRT we do not have a precisely defined muon track energy. If we require a full passage through the stack (S1 counter), we can conclude only that the muon energy is higher than $1.6 \mathrm{GeV}$. Furthermore, as can be seen in Fig. 12, which shows the Cherenkov angle resolution as a function of muon energy, the CRT stack thickness is not optimum, and the Cherenkov angle resolution has a large contribution from multiple scattering for soft tracks.

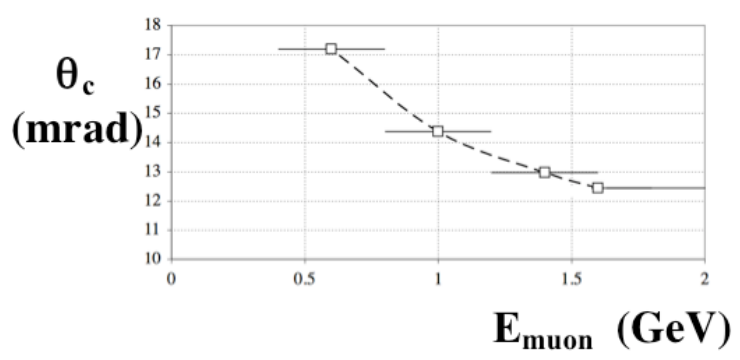

Fig.12. Measured Cherenkov angle resolution as a function of muon energy, which is determined from the muon depth in the iron stack using the S1-S4 counters in the CRT. The rise in resolution at low energy is explained by multiple scattering.

We will now investigate the tails of the Cherenkov angle distribution. Figures 10 and 11a show clearly that tails are larger in the case of 3D tracks than when tracks are perpendicular to the bar. Tails in the Cherenkov angle distribution are caused by ambiguities and are more significant for 3D tracks. In this prototype we have only two ambiguities ${ }^{6}$ : we cannot tell the sign of $\mathrm{k}_{\mathrm{x}}$ as the photon leaves the bar end, and therefore in the analysis we had to consider both signs. One solution yields the correct Cherenkov angle and the other contributes to the tail. We refer to this effect as the left-right ambiguity. In principle there are also sign ambiguities in $\mathrm{k}_{\mathrm{y}}$ and $\mathrm{k}_{\mathrm{z}}$. However, we know the direction of the muon (vertically downwards), and we fix the sign of $\mathrm{k}_{\mathrm{z}}$ using the TOP variable, as forward and backward propagating photons are clearly resolved - see Fig. 6b. This

\footnotetext{
${ }^{6}$ In this FDIRC prototype design there is no wedge and the photon camera does not have reflecting sides.
}

prototype has only six $\mathrm{H}-8500$ detectors so that, when the dip angle exceeds $\sim 6^{\circ}$, the Cherenkov ring moves away from the detector acceptance, and we collect only background hits, thus reducing the $\mathrm{S} / \mathrm{N}$ ratio. This is illustrated in Fig. 13, which shows the Cherenkov angle vs. the track dip angle (angle between track and the vertical y-axis) for CRT data.

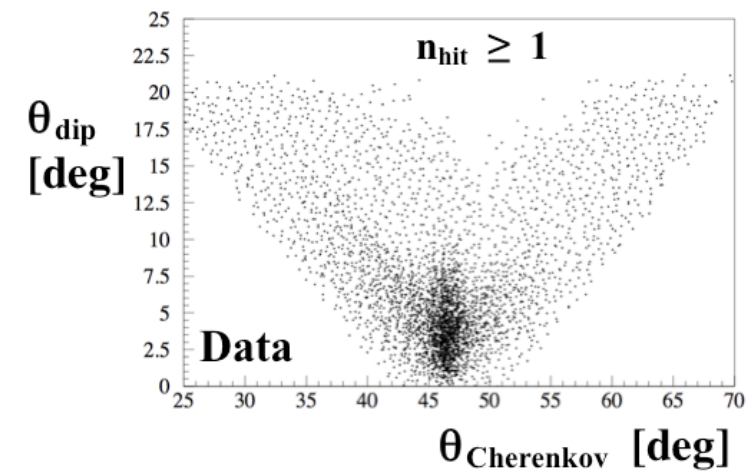

Fig.13. Measured Cherenkov angle $\theta_{\mathrm{c}}$ vs. the track dip angle using "reconstructed" photon pixel assignments, for $\mathrm{E}_{\text {muon }}>1.6 \mathrm{GeV}$ in the second FDIRC prototype in CRT.

Figure 14a shows that the corresponding $\mathrm{MC}$ simulation is in agreement with the data. Figure $14 \mathrm{~b}$ shows that the contribution from $\delta$-rays and scatters in the bar is relatively minor. We note that the $\mathrm{MC}$ has no contribution from showers which accompany some CRT muons. Finally, Fig. 14c shows a contribution from the left-right $\mathrm{k}_{\mathrm{x}}$ ambiguity from muon tracks only: this is by far the dominant contribution. Furthermore, this contribution tends to affect the resolution as some incorrect solutions are very close to the correct ones. In the present analysis each solution has equal weight.

When we run the MC program using "true" photon directions, there is no tail (these are photons generated at the source where direction cosines are known, which is possible only in MC simulation). We only observe the tail if we use the "reconstructed" photons (these are photons using photon vectors assigned to each pixel, i.e., a propagation all the way to a detector was involved). 

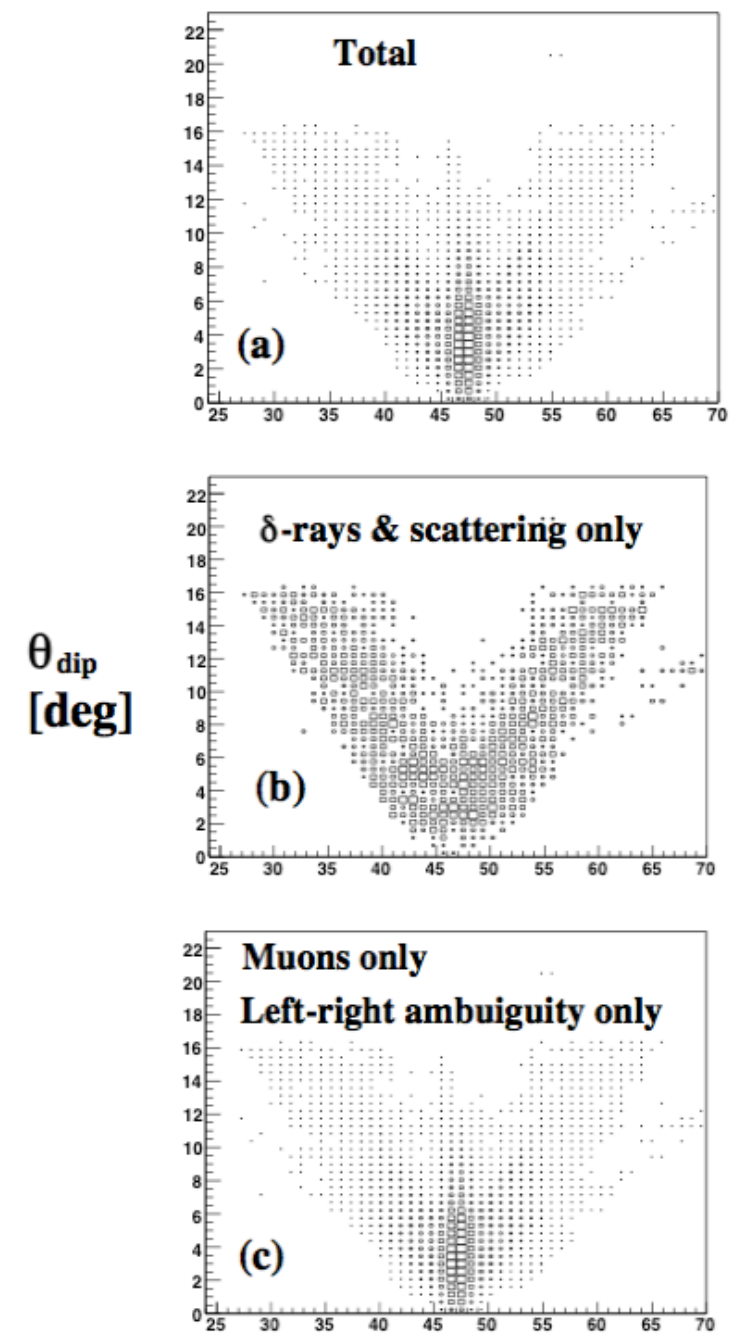

\section{$\theta_{\text {Cherenkov }}[\mathrm{deg}]$}

Fig.14. MC simulation of the Cherenkov angle $\theta_{\mathrm{c}}$ vs. the track dip angle for (a) all contributions, (b) hits only from $\delta$-rays and scattering in the bar (notice that this contribution is very small), and (c) contribution from muon signal-only and the left-right ambiguity in $\mathrm{k}_{\mathrm{x}}$.

In the remaining section we discuss possible practical methods to suppress the Cherenkov angle tail in the data analysis. Presently, we suppress tails by cutting out events with accompanying showers, recognized by multiple hits in the CRT hodoscopes. However, because the hodoscope size is relatively small, this does not guarantee that the photon camera does not get extra hits. We also remove non-physical photons propagating in the bar (either those with $\beta>1$, or a direction that contradicts the internal reflection law). We did not make cuts directly on the Cherenkov angle distribution, as this creates nonGaussian shapes. Instead we have investigated cuts on the photon TOP (time-of-propagation).

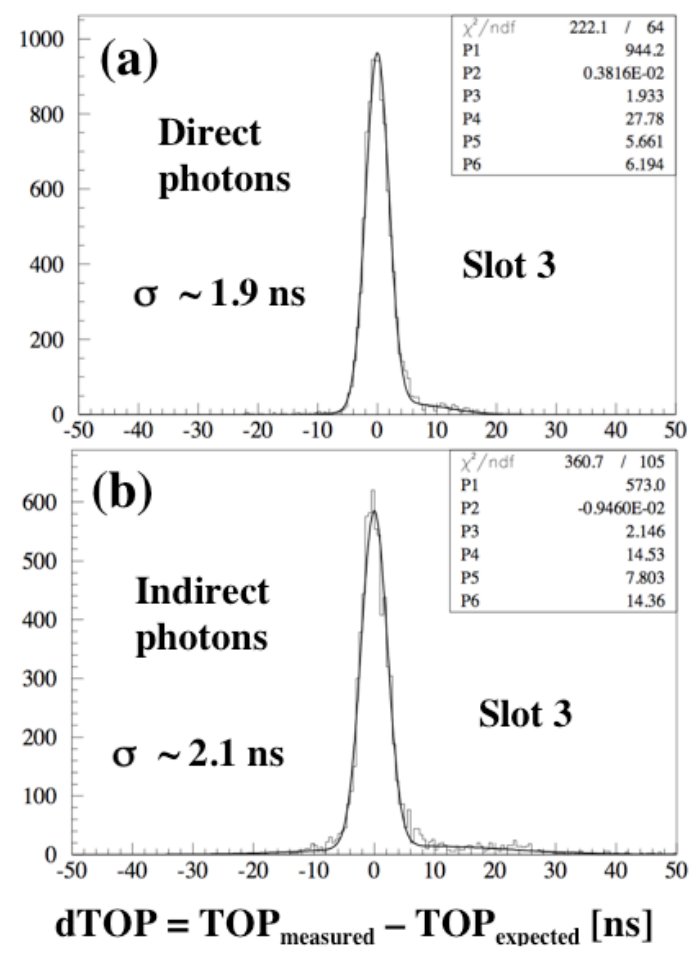

Fig.15. Measured $\mathrm{dTOP}=\mathrm{TOP}_{\text {measured }}-\mathrm{TOP}_{\text {expected }}$ distribution for slot 3 only, for $\mathrm{E}_{\text {muon }}>1.6 \mathrm{GeV}$ in the second FDIRC prototype in CRT.

Figure 15 shows the measured dTOP $=\mathrm{TOP}_{\text {measured }}$ $\mathrm{TOP}_{\text {expected }}$ distribution in this test in slot 3 for $3 \mathrm{D}$ tracks. One can see that we have achieved a dTOP timing resolution at a level of only $\sim 2 \mathrm{~ns}$. The direct photons $\left(\mathrm{k}_{\mathrm{z}}<0\right)$, which are generated propagating toward the camera, have slightly better resolution, and a smaller tail. However, this relatively poor timing resolution is limited by the BLAB2 electronics, as explained in section 4a. For comparison, Fig. 16 shows the dTOP performance of the first prototype in the SLAC test beam using the SLAC electronics [5-7] for the same bar position but with beam entering perpendicular to the bar face. In that configuration we achieved a dTOP timing resolution $\sigma \sim 210 \mathrm{ps}$ for direct photons, averaging 
over all slots; the indirect photon timing resolution is somewhat worse because of the chromatic broadening. However, the poor timing resolution of dTOP in this test does not affect the conclusions of this paper.

Figure 15 also shows that there is a rather small tail in the dTOP distribution, indicating that the background level due to showers accompanying cosmic ray muons is relatively small. Therefore we do not expect much benefit from the dTOP cut; this is shown on Fig. 17, where we plot the Cherenkov angle as a function of a cut in the dTOP variable; we see a rather small improvement by making such cuts compared to Fig. 11a, which does not include any cut on dTOP.
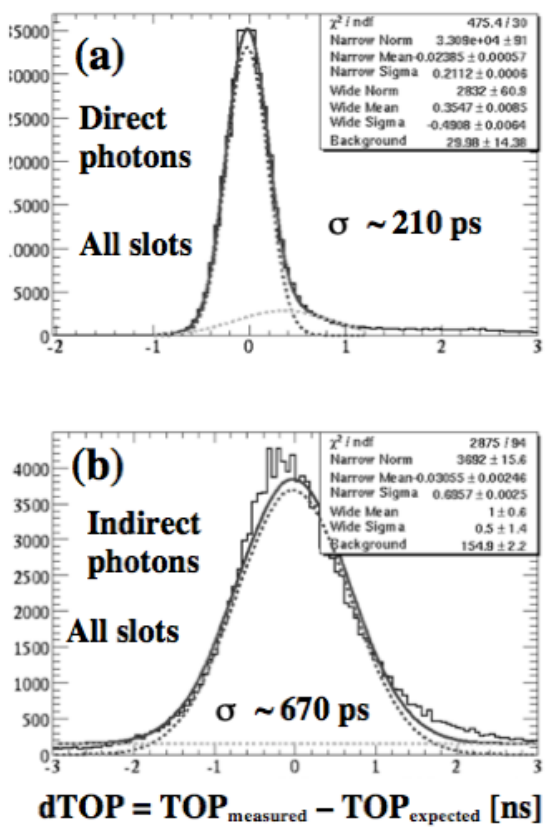

Fig.16. Measured of dTOP $=\mathrm{TOP}_{\text {measured }}-\mathrm{TOP}_{\text {expected }}$ distribution in the first FDIRC prototype in the test beam for all slots in the beam test of the same FDIRC prototype using the SLAC electronics $[5,6,7]$.

Figure 18 shows a plot of Cherenkov angle as a function of number of photon bounces in the bar. We see that the Cherenkov resolution is the best for $\mathrm{n}_{\mathrm{x}}<10 \quad(\sigma \sim 10.4 \mathrm{mrad})$, and worst for $\mathrm{n}_{\mathrm{x}}>400$ $(\sigma \sim 18.3 \mathrm{mrad})$. Figure $8 \mathrm{c}$ shows that $\mathrm{n}_{\mathrm{x}}<10$ corresponds to the central portion of the Cherenkov ring, and $\mathrm{n}_{\mathrm{x}}>400$ corresponds to its wing region.
This optical aberration was first realized in [5], and later further studied for several mirror types in [16]. Figure 19 summarizes the conclusions: a square bar alone introduces the optical aberration in the ring even for a simple pin hole focusing, but the effect is relatively small (Fig. 19d), and; a mirror amplifies this effect, especially near the ring wings. This effect depends very little on the type of mirror. Although the Cherenkov resolution is worse near its wings, we can still use these photons in the analysis, but with an appropriate weighting scheme.
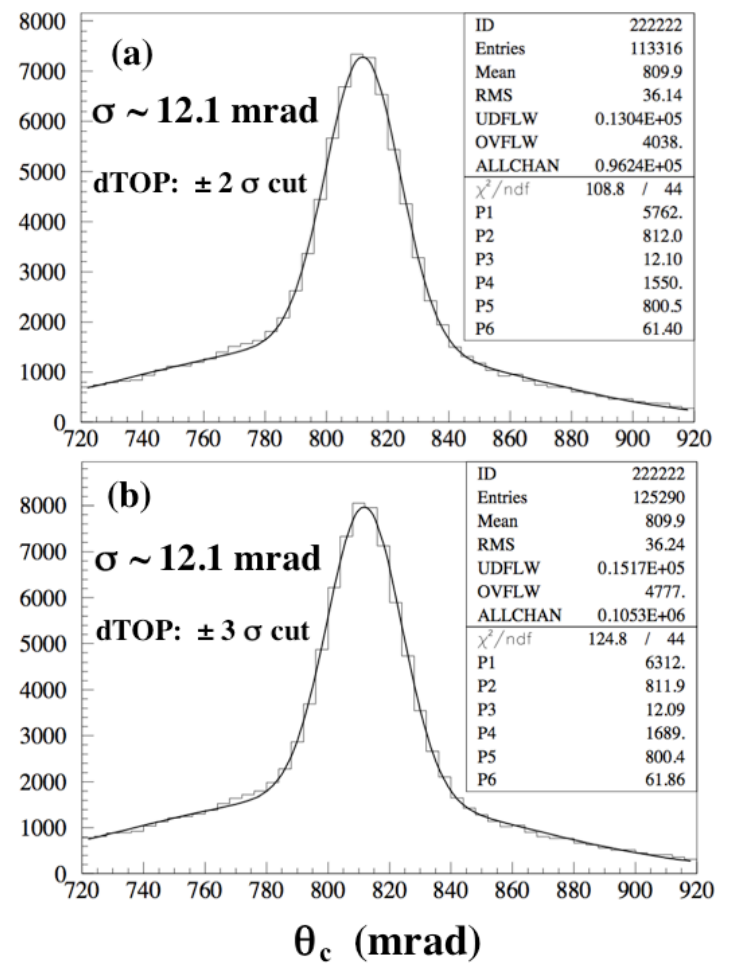

Fig.17. Measured Cherenkov angle as a function of cuts of $\mathrm{dTOP}=\mathrm{TOP}_{\text {measured }}-\mathrm{TOP}_{\text {expected }} \quad\left(\sigma_{\mathrm{TOP}} \sim 2 \mathrm{~ns}-\right.$ see Fig. 15). Note: the $\mathrm{S} / \mathrm{N}$ ratio does not change much if the dTOP cut is $\pm 1.5 \sigma$. 

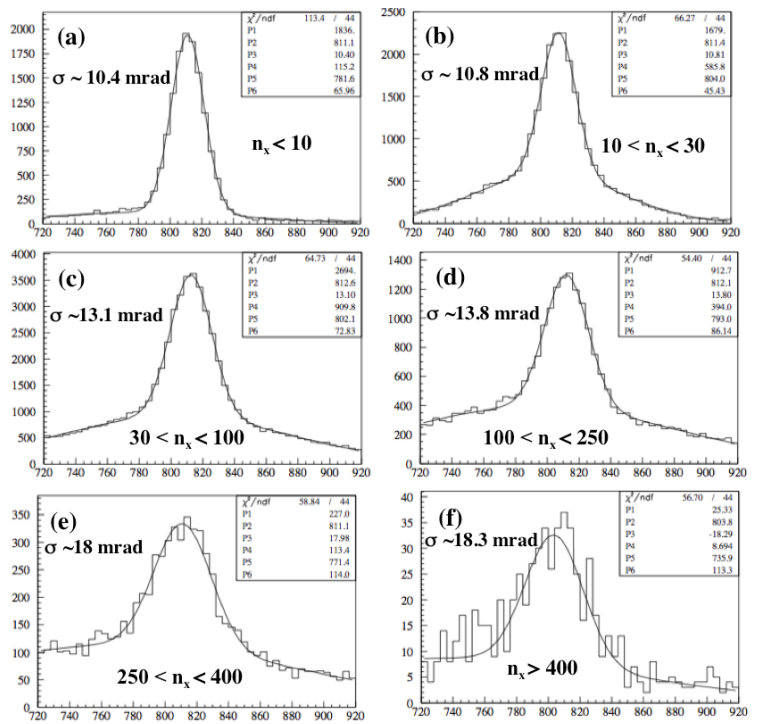

\section{$\theta_{\mathrm{c}}$ (mrad)}

Fig.18 Measured Cherenkov angle resolution for $\mathrm{E}_{\text {muon }}>$ $1.6 \mathrm{GeV}$ in the second FDIRC prototype for various slices in number of photon bar bounces in $\mathrm{x}$-direction $\mathrm{n}_{\mathrm{x}}$.
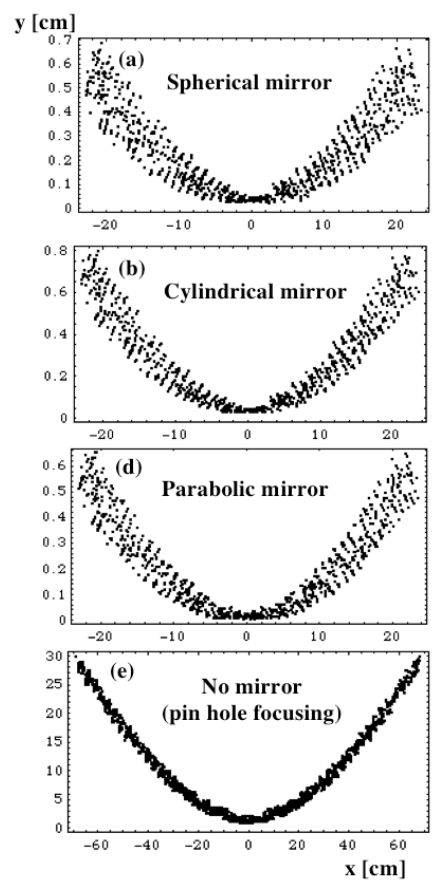

Fig.19 Simulated images with Mathematica [16] for several photon camera mirror configurations: (a) Spherical mirror, (b) cylindrical mirror, (c) parabolic mirror and (d) no mirror (pin hole focusing with a flat detector $50 \mathrm{~cm}$ away).
Figure 20 shows the measured Cherenkov angle $\theta_{\mathrm{c}}$ resolution for hard muons $(>1.6 \mathrm{GeV})$ for either (a) direct photons with $\mathrm{k}_{\mathrm{z}}<0$ and $\mathrm{n}_{\mathrm{x}}<10$ (from Fig. $8 \mathrm{~b}$ this range of $\mathrm{n}_{\mathrm{x}}$ values corresponds to a range of $100<\mathrm{n}_{\mathrm{y}}<170$ ), or (b) indirect photons with $\mathrm{k}_{\mathrm{z}}>0$ and $\mathrm{n}_{\mathrm{x}}<10$ (from Fig.8b this range of $\mathrm{n}_{\mathrm{x}}$ values corresponds to a range of $400<\mathrm{n}_{\mathrm{y}}<530$ ). The Cherenkov angle resolution is very good for these conditions, which correspond to the very central portion of the Cherenkov ring as one has the least optical aberration here. This reinforces our explanation based on the kaleidoscopic effect shown on Fig. 19a (the first and second FDIRC prototypes used a spherical mirror, and the final FDIRC prototype will use a cylindrical mirror). We also observe that the resolution is about the same for both forward and backward photons, indicating that we are not sensitive to a very large number of bounces in the y-direction, which are bounces from bar faces see Fig. 20b. This indicates that the bar surfaces are very flat and parallel.
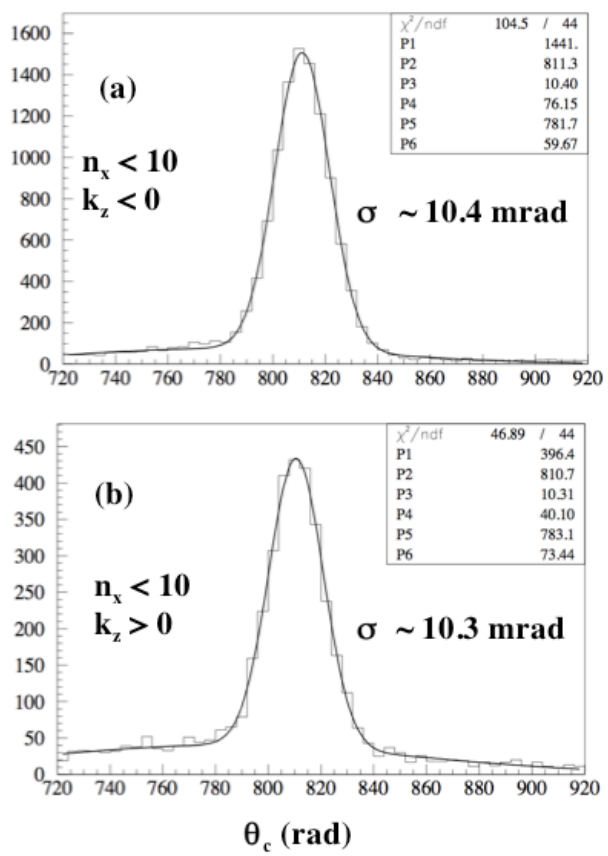

Fig.20 Measured Cherenkov angle resolution for $\mathrm{E}_{\text {muon }}>1.6 \mathrm{GeV}$ in the second FDIRC prototype in CRT for a small number of bounces in $\mathrm{x}$-direction $\left(\mathrm{n}_{\mathrm{x}}<10\right)$, and for either (a) direct photons $\left(\mathrm{k}_{\mathrm{z}}<0\right)$, or $(\mathrm{b})$ indirect photons $\left(\mathrm{k}_{\mathrm{z}}>0\right)$. 


\section{Conclusions}

This paper presents a detailed study of Cherenkov rings in the second FDIRC prototype. We show that the CRT setup is very useful to perform detailed studies with 3D tracks of the FDIRC optics and to develop analysis strategies. The 3D aspect of the analysis discovered new features of the FDIRC detector, which can be summarized as follows:

1. The major result of this paper is that the main contribution to the Cherenkov angle tail is coming from left-right ambiguities in $\mathrm{k}_{\mathrm{x}}$. In this paper we have treated both signs of $\mathrm{k}_{\mathrm{x}}$, with equal weight. In the final FDIRC prototype we will try to develop a weighting algorithm to suppress less statistically likely solutions.

2. From equation (4) it is clear that TOP depends only on $\mathrm{k}_{\mathrm{z}}$, and not on $\mathrm{k}_{\mathrm{x}}$. Therefore the $\mathrm{k}_{\mathrm{x}}$ ambiguity cannot be resolved by timing alone.

3. We clearly show that the best Cherenkov angle resolution is obtained using the Cherenkov ring's central region for FDIRC designs with mirrors, and the least precise portion is in the Cherenkov ring wings. This point will be investigated in the final FDIRC analysis by trying to assign a proper weight to each photon in the (x,y, time)-space.

This study will feed into development of the optimum analysis strategy for the final FDIRC prototype [17], which is currently being completed.

\section{Acknowledgements}

We would like to thank M. McCulloch for help in preparing various setups. This work has been supported in part by the Department of Energy Advanced Detector Research Program Award \# DEFG02-08ER41571.

\section{References}

[1] I. Adam, et al., Nucl. Instr. \& Meth. A 538 (2005) 281.

[2] B.N. Ratcliff, Nucl. Instr. \& Meth. A 502 (2003) 211.

[3] C. Field, T. Hadig, D.W.G.S. Leith, G. Mazaheri, B. Ratcliff, J. Schwiening, J. Uher, J. Va'vra, Nucl. Instr. and Meth. A 553 (2005) 96.

[4] C. Field, T. Hadig, M. Jain, D.W.G.S. Leith, G. Mazaheri, B. Ratcliff, J. Schwiening, J. Va'vra, Nucl. Instr. \& Meth. A 518 (2004) 565.
[5] J.F. Benitez, I. Bedajanek, D.W.G.S. Leith, G. Mazaheri, B. Ratcliff, K. Suzuki, J. Schwiening, J. Uher, J. Va'vra, Development of a Focusing DIRC, IEEE Nuclear Science Conference Record, October 29, SLAC-PUB12236, 2006.

[6] J. Va'vra, J.F. Benitez, I. Bedajanek, D.W.G.S. Leith, G. Mazaheri, B. Ratcliff, K. Suzuki, J. Schwiening, J. Uher, The Focusing DIRC - the First RICH Detector to Correct the Chromatic Error by Timing, Presented at Vienna Conference on Instrumentation, February, SLACPUB-12803, 2007.

[7] J.F. Benitez, I. Bedajanek, D.W.G.S. Leith, G. Mazaheri, B. Ratcliff, L.L. Ruckman, K. Suzuki, J. Schwiening, J. Uher, G.S. Varner, J. Va'vra, Nucl. Instr. \& Meth. A 595 (2008) 104.

[8] L.L. Ruckman, K. Nishimura, G.S. Varner, J. Va'vra, D. Aston, D.W.G.S. Leith, B. Ratcliff, Nucl. Instr. \& Meth. A 623 (2010) 303.

[9] G.S. Varner, L.L. Ruckman, J. Schwiening, J. Va'vra, Compact, Low-power and Precision Timing Photodetector Readout, in: Proceedings of the Photo-Detector 2007 Conference, July, Kobe, Japan, Proceedings of Science, PoS (PD07), 2007, p. 026.

[10] G.S. Varner, L.L. Ruckman, J.W. Nam, R.J. Nichol, J. Cao, P.W. Gorham, M. Wilcox, Nucl. Instr. \& Meth. A 583 (2007) 447.

[11] L. Ruckman, G. Varner, A. Wong, Nucl. Instr. \& Meth. A 591 (2008) 534.

[12] L.L. Ruckman, G.S. Varner, Nucl. Instr. \& Meth. A 602 (2009) 438

[13] L.L. Ruckman, Master of Science Thesis, University of Hawaii, May, 2010.

[14] A. Yarritu, S. Spanier, J. Va'vra, SLAC-PUB-9073, Nov. 1, 2001, and BaBar DIRC note \#141, Feb. 19, 2003.

[15] J. Va'vra, SLAC-PUB-13873, January 8, 2010.

[16] J. Va'vra, "Simulation of the Focusing DIRC Optics with Mathematica," SLAC-PUB-13464, 2008.

[17] J. Va'vra, D. Roberts and B. Ratcliff, Nucl. Instr. \& Meth. A 639 (2011) 282. 